\title{
Gastrodin provides neuroprotection in models of traumatic brain injury via Nrf2 signaling pathway
}

\author{
Dan Wang ${ }^{1}$, Xiaoqing Dong ${ }^{2, *}$ \\ ${ }^{1}$ Department of Neurology, Qingyang People's Hospital, Qingyang, Gansu, China; ${ }^{2}$ Department of Neurosurgery, Daye \\ People's Hospital of Hubei Province, Daye, Hubei, China
}

"Corresponding Author: Xiaoqing Dong, Department of Neurosurgery, Daye People's Hospital of Hubei Province, No. 25 Dongfeng Road, Jōhoku Development Zone, Daye, Hubei, China. Email: dongxiaoqing74@163.com

Received: 14 September 2021; Accepted: 24 November 2021; Published: 7 December 2021

(c) 2021 Codon Publications

OPEN ACCESS CC) ORIGINAL ARTICLE

\begin{abstract}
Gastrodin is one of the main active components of Gastrodia elata and has significant therapeutic value for various nervous system diseases. Its medicinal properties include smooth muscle relaxation, anti-necrosis, anti-aging, and anti-apoptosis effects. However, its possible effects on traumatic brain injury (TBI) are still unclear. In this study, the effects of gastroditin on TBI rats were investigated. The results proved that gastrodin had neuroprotective effect on TBI through alleviating brain deficits, decreasing brain water content, inhibiting neuronal apoptosis, and suppressing oxidative stress in brain tissues of TBI rats. Mechanically, gastrodin upregulated the expression of Nrf2 downstream proteins, suggesting the activation of Nrf2 pathway in brain tissues of TBI rats. In conclusion, gastrodin provided neuroprotection in TBI rats via Nrf2 pathway.
\end{abstract}

Keywords: apoptosis; gastrodin; Nrf2 pathway; oxidative stress; traumatic brain injury (TBI)

\section{Introduction}

Traumatic brain injury (TBI) is a common injury in both peacetime and wartime (Schmitt et al., 2021). It is estimated that there are about 10 million TBI patients worldwide each year, with a high prevalence of mild TBI (mTBI), accounting for about $76 \%-83 \%$. The pathophysiological mechanisms of TBI are excitatory toxic injury, inflammation, increased vascular permeability, and oxidative stress (Suntai et al., 2021). The series of pathological changes further aggravate the secondary brain damage caused by TBI, which is characterized by oxidative stress and inflammatory processes (Huibregtse et al., 2021). Depletion of antioxidant enzymes could lead to excessive production of reactive oxygen species (ROS), which might destroy cellular components such as protein, lipid, and DNA, thus exacerbating neuronal apoptosis (Thomas et al, 2021). Brain dysfunction after TBI is not only due to the original external mechanical forces, such as the role of primary injury, but also to a large extent related to the "secondary attack" of neurons complicated by the injury, called secondary neuronal injury, whose mechanisms mainly include calcium overload, excitability amino acid toxic effects, mitochondrial dysfunction, oxidative stress, etc. Therefore, it is very important to find an effective method to reduce the oxidative stress caused by TBI.

Gastrodin is one of the main active components of Gastrodia elata, which has been isolated and confirmed as a traditional Chinese herbal medicine (Qin et al., 2021). Gastroditin is the main bioactive ingredient from the rhizome of Gastrodia elata, and has significant therapeutic value for nervous system diseases (Yao et al., 2019). In addition, its medicinal properties include smooth muscle relaxation, anti-necrosis, anti-aging, and anti-apoptotic effects (Nepal et al., 2019). Gastrodin could effectively reduce the level of lipid peroxidation, remove oxygen-free radicals, play an antioxidant role, reduce coupled oxidative phosphorylation, and increase the levels of malondialdehyde and superoxide deaminase (Li et al., 2019). In addition, gastrodin could improve cell 
pyroptosis caused by microvascular reperfusion injury via mediating the NLRP3 pathway (de Oliveira et al., 2019). However, the possible effects of gastrodin on TBI, an inflammation-related disease, were still unclear.

The Nrf2 signaling pathway plays a key role in neuroprotection. Astrocytes and neurons of primary cultured $\mathrm{Nrf2}^{-1-}$ mice were more susceptible to oxidative damage, calcium disorder, and mitochondrial toxicity than wildtype cells (de Oliveira et al., 2019; Hwang et al., 2019). Hwang et al. (2019) found that nerve cells pretreated with coffee white fat could activate Nrf2 and its downstream HO-1, and protect nerve cells from Parkinson's related neurotoxin 6-hydroxy dopamine (6-OHDA). It is therefore hypothesized that Nrf2 pathway has potential effects on the inflammation in several diseases. Targeting this signal pathway is important for the treatment of related neurological diseases.

In this study, the effects of gastroditin on a rat model of TBI were investigated. The investigations showed that gastrodin inhibited neuronal apoptosis and suppressed oxidative stress in brain tissues in TBI-induced rats. Gastrodin could serve as a promising and potential drug for the treatment of TBI.

\section{Materials and Methods}

\section{Traumatic brain injury (TBI) rat model}

Sprague-Dawley (SD) rats (Jackson Laboratories, ME, USA) were subjected to TBI model construction. Rats were fed ad libitum and kept under a 12-h/12-h light-dark cycle. All the animal experiments were performed with the approval of the Institutional Animal Care and Use Committee of Qingyang People's Hospital (Gansu, China) (Approval no. 2019141). Chloral hydrate $(5 \%, 0.1 \mathrm{~mL} / 10 \mathrm{~g})$ was used for anesthesia. Rats were fixed to the stereotaxic apparatus, and the skull was widely exposed. TBI model was constructed with 450-g steel weight dropping from $1.5 \mathrm{~cm}$ height. Thus, the left cortex was moderately injured. The animals in the sham group were subjected to the same operation except for the dropping of the steel weight. Gastrodin (purity $>99.2 \%$ ) was purchased from Kunming Pharmaceutical Corp (Kunming, Yunnan, China). In TBI+ gastrodin group, after the operation, rats were administrated with the indicated concentration of gastrodin for a week. Thereafter, rats were sacrificed by breaking the neck for further study. Rats in the sham group underwent the same surgery.

\section{Neurobehavioral evaluation}

The neurological score was evaluated with a well-established, neurological severity scoring (NSS), open-field and Rotarod testing after TBI. The evaluation was carried out without knowing the different treatments. Behavioral tests were repeatedly performed to further validate the outcomes. The NSS test reflects the motor (muscle status, abnormal movement), sensory (visual, tactile, and proprioceptive), balance, and reflex functions of rats. Neurological score ranging from 0 to $18(0=$ normal function; 18 =maximal deficit) represents the neurological lesion.

\section{Measurement of brain water content}

The brain in rats was removed immediately after sacrifice. Then, the brain weight was recorded and then dried in an oven until the weight was unchanged. The dried brain was weighed to get dry weight. The percentage of brain water was calculated as [(Wet weight-Dry weight)/ Wet weight] ${ }^{*} 100 \%$.

\section{TUNEL staining}

Sliced sections were digested with $20 \mathrm{mg} / \mathrm{mL}$ proteinase $\mathrm{K}$ at $37^{\circ} \mathrm{C}$ for $15 \mathrm{~min}$. Then, sections were rinsed in phosphate-buffered saline (PBS) and added with $0.3 \% \mathrm{H}_{2} \mathrm{O}_{2}$ for $10 \mathrm{~min}$, followed by incubation with $0.1 \%$ sodium citrate and $0.1 \%$ Triton X-100 solution for 2 min. TUNEL reaction mixture with terminal deoxynucleotidyltransferase (TdT) (Sigma-Aldrich, St. Louis, MO, USA) was added into the sections at $37^{\circ} \mathrm{C}$ under humidified conditions followed by 4',6-diamidino-2-phenylindole (DAPI) staining. Each image was captured under a confocal microscope.

\section{Assessment of antioxidant activity}

The levels of superoxide dismutase (SOD), catalase $(\mathrm{CAT})$, glutathione peroxidase (GSH-Px), and malondialdehyde (MDA) were spectrophotometrically determined with the commercial kits from Nanjing Jiancheng Bioengineering Institute (Jiangsu, China) in accordance with manufacturer's instructions.

\section{Western blot analysis}

Brain tissues were homogenized with RIPA lysis buffer (Beyotime Institute of Biotechnology, Jiangsu, China). Supernatants were obtained by centrifugation of homogenates at $12,000 \times \mathrm{g}$ at $4{ }^{\circ} \mathrm{C}$ for $10 \mathrm{~min}$. After the protein concentration was measured by BCA Protein Assay Kit, protein samples were electrophoresed and transferred onto PVDF membranes (EMD Millipore, Billerica, MA, USA). Then, the membranes were blocked with $5 \%$ 
bovine serum albumin for $1 \mathrm{~h}$, and incubated with the primary antibodies: GAPDH (1:10000; ab8245, Abcam, Cambridge, UK), BCL-2 (1: 1,000; ab32124, Abcam), Bax (1: 1,000; ab32503, Abcam), Cleaved-caspase 3 (1: 1,000; ab32042, Abcam), Nrf2 (1:1000; ab62352, Abcam), H3 (1:1000; ab1791, Abcam), HO-1 (1:1000; ab52947, Abcam), and NQO-1 (1:1000; ab80588, Abcam) for $2 \mathrm{~h}$ at room temperature. Subsequently, the membranes were incubated with anti-rabbit or anti-mouse IgG, HRPlinked antibodies at room temperature for $1 \mathrm{~h}$. The blots were analyzed with an ECL kit (Abcam).

\section{Statistical analysis}

Results were displayed as mean \pm SD. Statistical analysis was performed using GraphPad Prism 5.03 (GraphPad Software Inc., San Diego, CA, USA). Significance was assessed by Student's t-test. $p<0.05$ was considered as a significant value.

\section{Results}

\section{Gastrodin exerts neuroprotective effects on TBI rats}

To evaluate the effects of gastrodin on brain deficits in TBI rats, the NSS score in TBI rats after gastrodin treatment was analyzed. As shown in Figure 1(A), compared to rats in the sham group, TBI caused a significantly higher NSS score. The NSS score was reduced in gastrodin-treated TBI rats. Moreover, the water content in the brain was also assessed. TBI stimulation induced elevated brain water content compared to rats in the sham group. However, gastrodin reduced water content in TBI rats (Figure 1B). The results indicated that gastrodin promoted neural injure recovery after TBI establishment.

\section{Gastrodin inhibits the apoptosis of nerve cells in TBI rats}

The number of TUNEL-positive cells was calculated to evaluate the effects of gastrodin on the apoptosis of nerve cells. The TUNEL-positive cell number was elevated dramatically in the TBI group. However, significantly fewer TUNEL-positive cells were observed in gastrodin-treated TBI rats (Figure 2A). Additionally, the expression levels of the pro-apoptotic proteins Bax and BCL-2 were measured. TBI induced the protein expressions of Bax and cleaved caspase-3, and suppressed BCL-2 protein expression. However, gastrodin treatment markedly reversed these protein alterations (Figure 3B), suggesting its protective role against nerve cell apoptosis.

\section{Gastrodin alleviates TBI-induced oxidative stress}

To examine the effects of gastrodin on oxidative stress, the levels of MDA, GSH-px, SOD, and CAT were determined. The levels of SOD, GSH-px, and CAT in the TBIinduced group were significantly decreased, whereas that of MDA was markedly enhanced (Figure 3). Gastrodin greatly exerted the antioxidant capacity, evidenced by increasing the levels of SOD, GSH-px, and CAT, and decreasing the MDA level (Figure 3). These data suggested that gastrodin exerted antioxidative properties in TBI-treated rats.

\section{Gastrodin exerts neuroprotective effects in TBI rats via Nrf2 signaling pathway}

To reveal the potential mechanism of gastrodin-mediated effect in TBI rats, the regulatory role of gastrodin in Nrf2 signaling pathway was determined. The Nrf2 signaling pathway was activated in TBI rats, as demonstrated
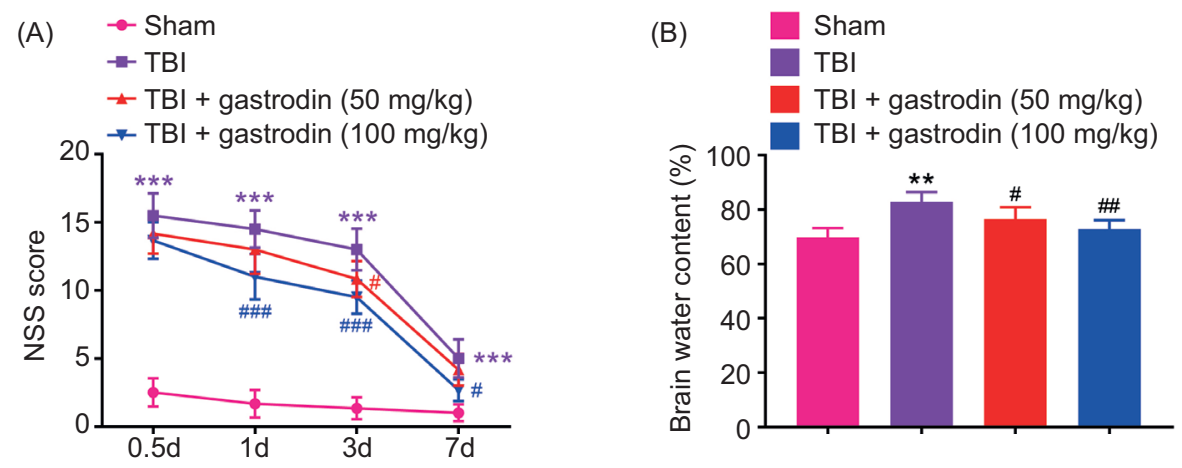

Figure 1. Gastrodin alleviates brain injury in TBI rats. (A) The NSS score of brain in the sham, TBI, TBI + gastrodin 50 and 100 $\mathrm{mg} / \mathrm{kg}$ groups. (B) The brain water content in the sham, TBI, TBI + gastrodin 50 and $100 \mathrm{mg} / \mathrm{kg}$ groups. ${ }^{* *} p<0.01,{ }^{* * *} p<0.001$ versus sham group; $\# p<0.05,{ }^{\prime} p<0.01, \ldots+0.001$ versus TBI group. 


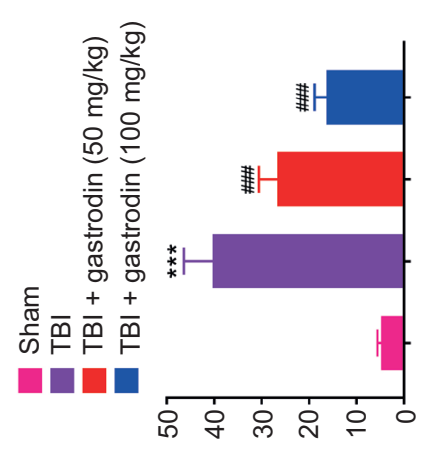

(\%) s!soldod $\forall$

$\widehat{\underline{m}}$
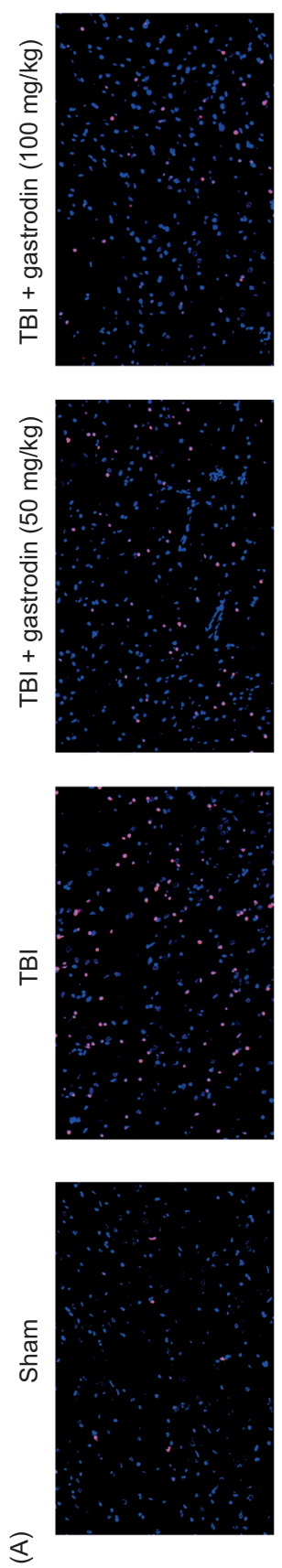

$\stackrel{0}{=}$

鱼

ํㅡㅁ

흔

कำ

뉴

응 웅

호 $v$

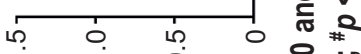

ио!़səлdxə $\varepsilon$-əsedseว -рәлеәј әм!ฺе|әу

을

등 흥

站

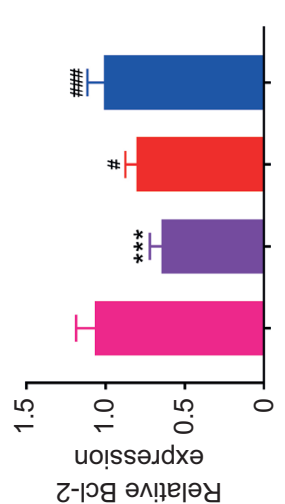

$+$

$\overline{\mathrm{m}} \stackrel{0}{\mathrm{~m}}$

家

몬

हิ

$\frac{\pi}{4}$ v

视絭

든 ํํㅇ

흠

흥

응

옫

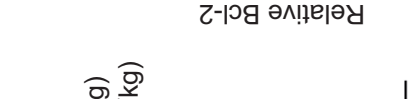

응

क⿺辶万

듕

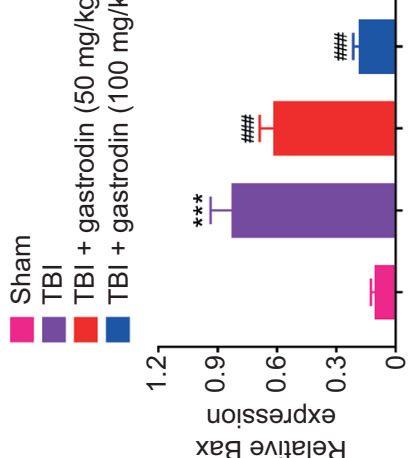

는

드.

을 은

区

혼

商

商

高 $\frac{\varepsilon}{\frac{\pi}{5}}$

xeg ә시리맘

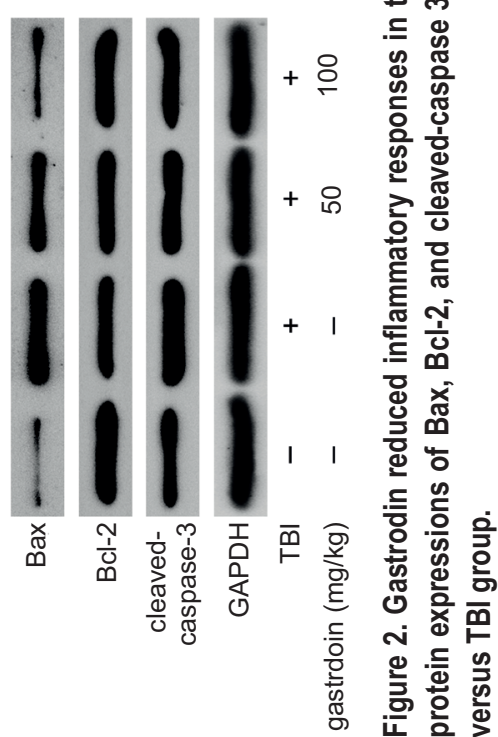



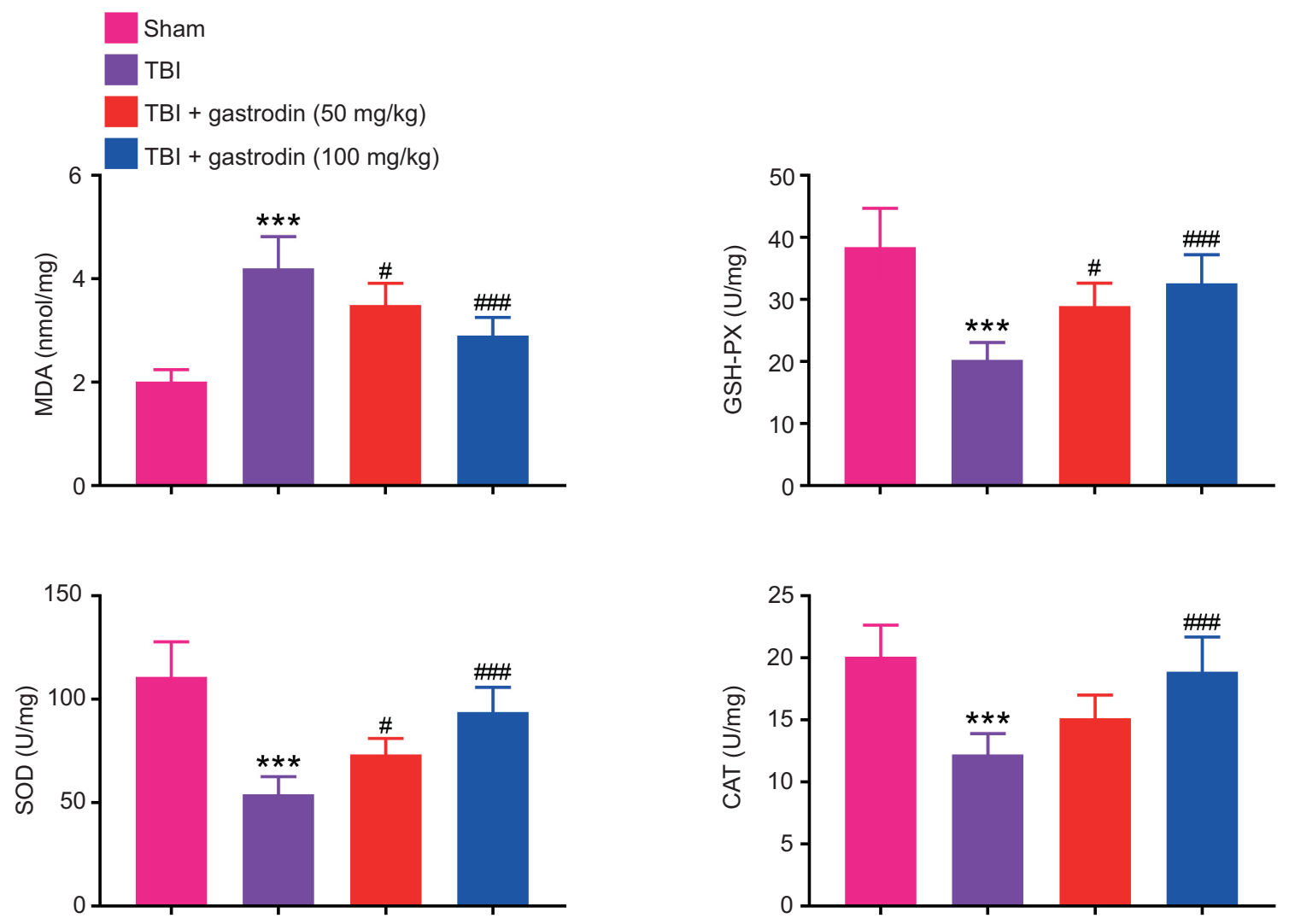

Figure 3. Gastrodin repressed oxidative stress in the brain of TBI rats. The levels of MDA, GSH-px, SOD, and CAT in the brain of sham, TBI, TBI + gastrodin 50 and $100 \mathrm{mg} / \mathrm{kg}$ groups. ${ }^{* * *} p<0.001$ versus sham group; ${ }^{\#} p<0.05,{ }^{* \#} p<0.001$ versus TBI group.

by increased levels of Nrf2, HO-1, and NQO-1. The increased levels of Nrf2, HO-1, and NQO-1 were further aggravated by gastrodin treatment (Figure 4). These data suggested that gastrodin exerts neuroprotective effects on TBI rats via Nrf2 signaling pathway.

\section{Discussion}

The incidence of TBI has been increasing in recent years. Despite the great progress in research on TBI and improvements in diagnostic techniques and treatment measures, its mortality and disability rates have not decreased significantly (Wearne et al., 2021). Due to its complex diagnosis and treatment, high mortality and disability rates, its mechanism of injury has been one of the hot spots in neurosurgical research (Sowter et al., 2021). A large number of studies have confirmed that brain dysfunction after TBI is not only caused by the primary injury such as the initial mechanical force, but also largely related to the complex "secondary strike" of neurons, namely the secondary neuron injury (Merritt et al., 2021). In this study, gastrodin inhibited neuronal apoptosis and inhibited oxidative stress in brain tissues in TBI rats. The present data provided a new sight into the pathology of TBI and provided a promising drug for TBI treatment.

Oxidative stress refers to the process of excessive production of highly active molecules such as ROS in the body when the body is subjected to various harmful stimuli, and the imbalance between oxidation system and antioxidant system results in tissue damage (Zhang et al., 2019). After TBI occurs, a large number of free radicals are generated, and oxidative stress plays an important role in the pathophysiological process after TBI. Oxidative stress causes direct damage to nerve cells through lipid peroxidation and protein oxidation. In addition, it can also mediate mitochondria and other pathways to indirectly induce neuronal apoptosis (Yuan et al., 2019). Interestingly, in this study, gastrodin inhibited TBI-induced neuronal apoptosis in rats. In addition, gastrodin inhibited TBIinduced oxidative stress in brain tissues in rats. These data confirmed that this drug had a protective effect against neuronal apoptosis and oxidative stress, two important causes of TBI, proving its potential in treating TBI.

The various biological activities of gastrodin have been widely reported in previous studies. Gastrodin played an important role in maintaining normal neuron 

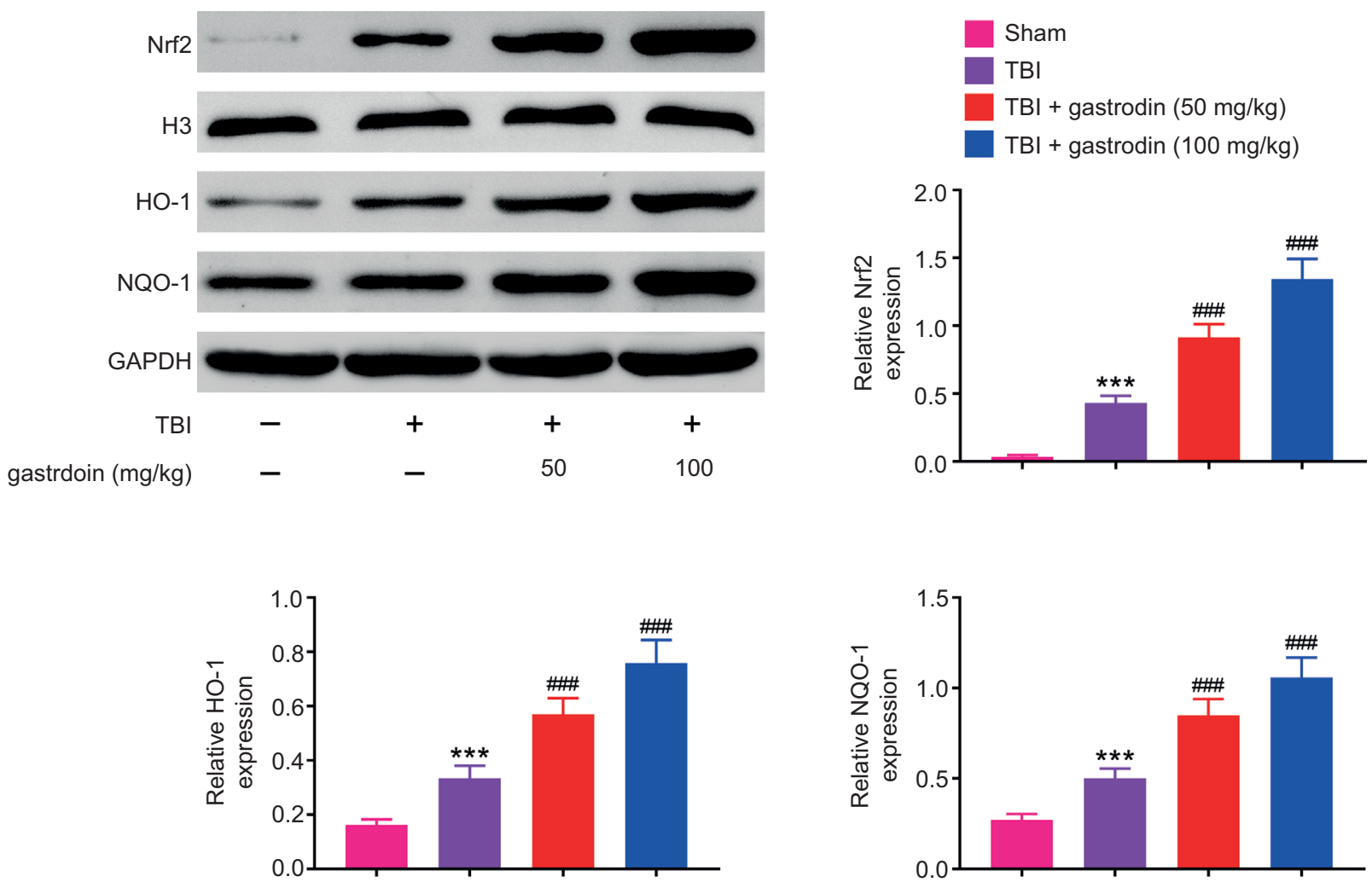

Figure 4. Gastrodin suppresses NLRP3 inflammasome signaling pathway in the brain of TBI rats. The protein expression levels of Nrf2, H3, HO-1, and NQO-1 in the brain of sham, TBI, TBI + gastrodin 50 and $100 \mathrm{mg} / \mathrm{kg}$ groups. ${ }^{* *} p<0.001$ versus sham group; $"$ " 0.001 versus TBI group.

function (Yang et al., 2020). For example, gastrodin promotes hippocampal neurogenesis in mice following cerebral ischemia via PDE9 pathway (Xiao et al., 2021). Gastrodin could attenuate inflammatory response and cell migration via Notch-1 pathway in activated microglia (Yao et al., 2021). In addition, in the nervous system, gastrodin alleviated the neurocognitive dysfunction of aged mice via suppressing neuroinflammation and promoted the myelination of the central nervous system (CNS) (Lv et al., 2020). Notably, a previous study indicated the nephroprotective effects of gastrodin against lead-induced oxidative stress and inflammation in mice, which was consistent with our findings. Gastrodin also has other biological functions. It could alleviate cerebral ischemia/reperfusion injury via inhibiting pyroptosis. Whether gastrodin could exert protective effects on TBI through its other abilities need further investigation.

In this study, it is noticed that gastrodin upregulated the expression of Nrf2 downstream proteins, suggesting the activation of Nrf2 pathway in brain tissues of TBI rats. The Nrf2 pathway plays a key role in neuroprotection (Chen et al., 2021). It has been reported that several drugs, including gypenosides, provide neuroprotection effects via Nrf2 pathway (Tian et al., 2021). Astrocytes and neurons of primary cultured $\mathrm{Nrf} 22^{-1-}$ mice were more susceptible to oxidative damage (Dong et al., 2021). These studies, together with our findings, confirmed that the Nrf2 pathway could be a promising target to improve the neuroprotection effects.

In conclusion, the present study found that gastrodin had a neuroprotective effect on rats with TBI by inhibiting neuronal apoptosis and suppressing oxidative stress. Mechanically, gastrodin activated the Nrf2 pathway in brain tissues from TBI rats. Therefore, gastrodin could serve as a promising drug for TBI treatment.

\section{Availability of data and materials}

All data generated or analyzed during this study are included in this published article.

\section{Competing Interests}

The authors state that there are no conflicts of interest to disclose. 


\section{Ethics Approval}

Ethical approval was obtained from the Ethics Committee of Qingyang People's Hospital (Approval no. 2019141).

\section{Contribution of Authors}

Both Dan Wang and Xiaoqing Dong designed the study and supervised the data collection. Dan Wang analyzed and interpreted the data, Xiaoqing Dong prepared the manuscript for publication and reviewed the draft manuscript. All authors have read and approved the manuscript.

\section{References}

Chen J, Huang Y, Hu X, Bian X, Nian S. Gastrodin prevents homocysteine-induced human umbilical vein endothelial cells injury via PI3K/Akt/eNOS and Nrf2/ARE pathway. J Cell Mol Med. 2021;25(1):345-57. https://doi.org/10.1111/jcmm.16073

de Oliveira MR, Brasil FB, Furstenau CR. Nrf2 mediates the anti-apoptotic and anti-inflammatory effects induced by gastrodin in hydrogen peroxide-treated SH-SY5Y cells. J Mol Neurosci. 2019;69(1):115-22. https://doi.org/10.1007/s12031-019-01339-3

Dong Z, Bian L, Wang YL, Sun LM. Gastrodin protects against high glucose-induced cardiomyocyte toxicity via GSK-3beta-mediated nuclear translocation of Nrf2. Human Exp Toxicol. 2021;40(9):1584-97. https://doi. org/10.1177/09603271211002885

Huibregtse ME, Bazarian JJ, Shultz SR, Kawata K. The biological significance and clinical utility of emerging blood biomarkers for traumatic brain injury. Neurosci Biobehav Rev. 2021;130:43347. https://doi.org/10.1016/j.neubiorev.2021.08.029

Li S, Bian L, Fu X, Ai Q, Sui Y, Zhang A, et al. Gastrodin pretreatment alleviates rat brain injury caused by cerebral ischemicreperfusion. Brain Res. 2019;1712:207-16. https://doi. org/10.1016/j.brainres.2019.02.006

Lv H, Liu Y, Zhang B, Zheng Y, Ji H, Li S. The improvement effect of gastrodin on LPS/GalN-induced fulminant hepatitis via inhibiting inflammation and apoptosis and restoring autophagy. Int Immunopharmacol. 2020;85:106627. https://doi.org/10.1016/j. intimp.2020.106627

Merritt VC, Lange RT, Lippa SM, Brickell TA, Soltis AR, Dalgard $\mathrm{CL}$, et al. Apolipoprotein e (APOE) epsilon4 genotype influences memory performance following remote traumatic brain injury in U.S. military service members and veterans. Brain Cogn. 2021;154:105790. https://doi.org/10.1016/j.bandc.2021.105790

Nepal MR, Jeong KS, Kim GH, Cha DH, Kang MJ, Kim JS, et al. Role of intestinal microbiota in metabolism of gastrodin in vitro and in vivo. Metabolites. 2019;9(4):69. https://doi.org/0.3390/ metabo9040069

Qin B, Luo N, Li Y, Gong D, Zheng J, Tan X, et al. Protective effect of gastrodin on peripheral neuropathy induced by anti-tumor treatment with vincristine in rat models. Drug Chemical Toxicol. 2021;44(1):84-91. https://doi.org/10.1080/01480545.20 18.1547739

Schmitt RR, Kaliyappan K, Muthaiah VPK, Ignatowski TA, Prasad $\mathrm{PN}$, Mahajan SD. Blast-induced injury responsive relative gene expression of traumatic brain injury biomarkers in human brain microvascular endothelial cells. Brain Res. 2021;1770:147642. https://doi.org/10.1016/j.brainres.2021.147642

Sowter N, King L, Calderbank A, Eccles FJR. Factors predicting first appointment attendance at a traumatic brain injury clinical neuropsychology outpatient clinic: a logistic regression analysis. Disabil Rehabil. 2021;Sep 6:1-6. https://doi.org/10.1080/096382 88.2021.1970254

Suntai Z, Laha-Walsh K, Albright DL. Effectiveness of remote interventions in improving caregiver stress outcomes for caregivers of people with traumatic brain injury. Rehab Psychol. 2021. https://doi.org/10.1037/rep0000402

Thomas BP, Tarumi T, Wang C, Zhu DC, Tomoto T, Munro Cullum $\mathrm{C}$, et al. Hippocampal and rostral anterior cingulate blood flow is associated with affective symptoms in chronic traumatic brain injury. Brain Res. 2021;1771:147631. https://doi.org/10.1016/j. brainres.2021.147631

Tian ZK, Zhang YJ, Feng ZJ, Jiang H, Cheng C, Sun JM, et al. Nephroprotective effect of gastrodin against lead-induced oxidative stress and inflammation in mice by the GSH, Trx, Nrf2 antioxidant system, and the HMGB1 pathway. Toxicol Res. 2021;10(2):249-63. https://doi.org/10.1093/toxres/tfab003

Wearne TA, Logan JA, Trimmer EM, Wilson E, Filipcikova M, Kornfeld E, et al. Regulating emotion following severe traumatic brain injury: a randomized controlled trial of heart-rate variability biofeedback training. Brain Inj. 2021;Sep 6:1-12. https://doi. org/10.1080/02699052.2021.1972337

Xiao H, Jiang Q, Qiu H, Wu K, Ma X, Yang J, et al. Gastrodin promotes hippocampal neurogenesis via PDE9-cGMP-PKG pathway in mice following cerebral ischemia. Neurochem Int. 2021;150:105171. https://doi.org/0.1016/j.neuint.2021.105171

Yang GM, Li L, Xue FL, Ma CL, Zeng XF, Zhao YN, et al. The potential role of PKA/CREB signaling pathway concerned with gastrodin administration on methamphetamine-induced conditioned place preference rats and SH-SY5Y cell line. Neurotoxicity research. 2020;37(4):926-35. https://doi. org/10.1007/s12640-019-00150-7

Yao YY, Bian LG, Yang P, Sui Y, Li R, Chen YL, et al. Gastrodin attenuates proliferation and inflammatory responses in activated microglia through Wnt/beta-catenin signaling pathway. Brain Res. 2019;1717:190-203. https://doi.org/10.1016/j.brainres.2019.04.025

Yao YY, Li R, Guo YJ, Zhao Y, Guo JZ, Ai QL, et al. Gastrodin attenuates lipopolysaccharide-induced inflammatory response and migration via the Notch-1 signaling pathway in activated microglia. Neuromolecular Med. 2021. https://doi.org/10.1007/ s12017-021-08671-1 [online ahead of print].

Yuan F, Xu X, Wu Y, Duan S, Wu H. Gastrodin ameliorates acute rejection via IRE1alpha/TRAF2/NF-kappaB in rats receiving liver allografts. BioMed Res Int. 2019;2019:9276831. https://doi. org/10.1155/2019/9276831 
Zhang HB, Yuan B, Huang HF, Qu SM, Yang SK. Gastrodin induced HO-1 and Nrf2 up-regulation to alleviate $\mathrm{H} 2 \mathrm{O} 2$-induced oxidative stress in mouse liver sinusoidal endothelial cells through p38 MAPK phosphorylation Braz J Med Biol Res (2018) 51(10): e7439. https://doi.org/10.1590/1414-431X20187439
Zhang HS, Liu MF, Ji XY, Jiang CR, Li ZL, OuYang B. Gastrodin combined with rhynchophylline inhibits cerebral ischaemiainduced inflammasome activation via upregulating miR21-5p and miR-331-5p. Life Sci. 2019;239:116935. https://doi. org/10.1016/j.lfs.2019.116935 